

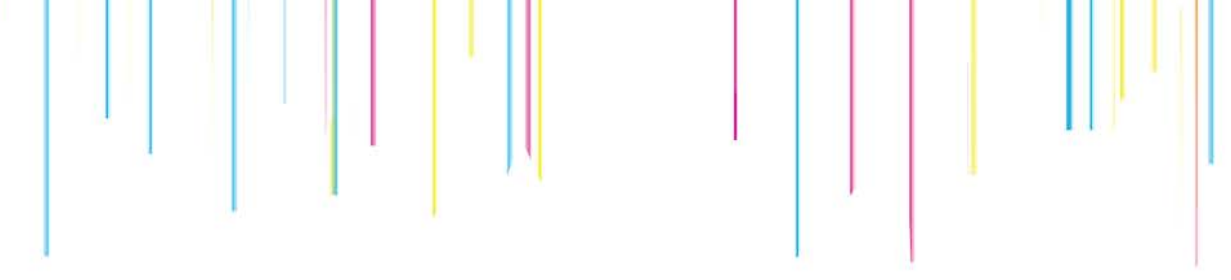

\section{The Otavalo Textile Grammar}

\subsection{Otavalo textiles}

Otavalo textiles may be categorized into three generalized pattern types as illustrated in Figure 2; axial, banded, and field. Axial textiles are often radial and display strong symmetry. Banded textiles are organized in stripes of various widths and designs. Fields are created through repetitive tiling of one or more geometric shapes. These pattern types can also be combined; axial patterns are often flanked by bands and have repeating elements, striped patterns can be organized symmetrically and incorporate radial configurations, tessellations are formed by regular figures and have an underlying logical structure.

Whether used to create geometrical patterns or figures such as mountains, plants, or animals, it is observed that many Otavalo textiles incorporate the same shape time and again: the isosceles trapezoid. A member of the quadrilateral family of shapes, the isosceles trapezoid is characterized by two parallel sides (making it a trapezoid) and symmetry (classifying it isosceles). Figure 3 demonstrates the recreation of a typical Otavalo textile using various sized and configured isosceles trapezoids.
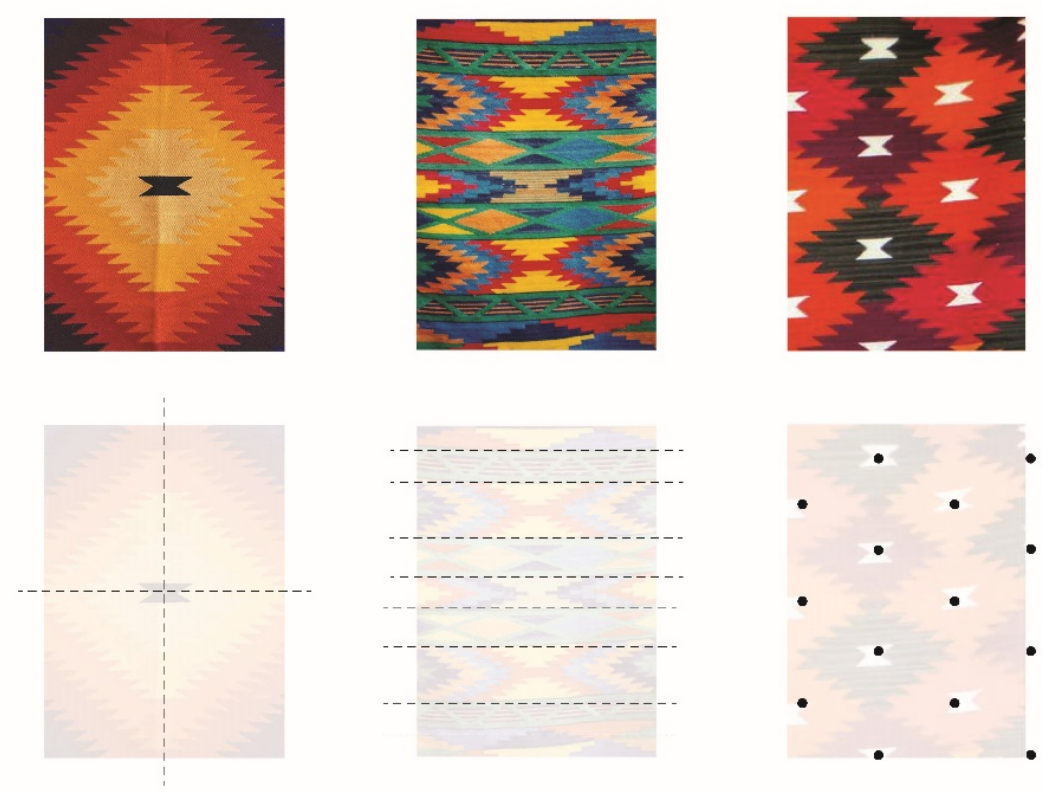

Figure 2. Otavalo textiles pattern types: axial (left, from Etsy, 2021), banded (center, from Fashion Pachanga, 2021), and field (right, from Pixabay, 2021). 

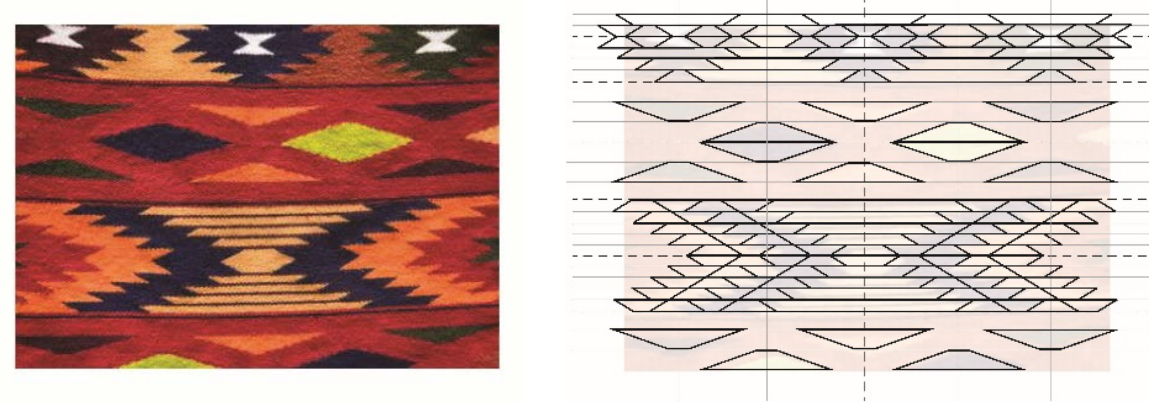

Figure 3. Recreation of an Otavalo textile pattern using isosceles trapezoids. (From Depositphotos, 2021).

\subsection{Shape definition}

A series of steps - illustrated in Figure 4 - define a parametric isosceles trapezoid.

a. An initial line of length " $x$ " is created between two points.

b. A copy of this line is translated by " $y$ " units.

c. A copy of the initial line is rotated by " $\theta$ " degrees about one of the end points, defining an intersection point between this angled line and the offset line.

d. The angled line is reflected about the center point of the initial line, defining another intersection point between this reflected line and the offset line.

e. A closed polyline is drawn between the four points.

f. This polyline defines an isosceles trapezoid surface.

A digital parametric model is created using the above steps, capable of producing a variety of isosceles trapezoids, controlled by three variables; length " $x$," height " $y$, , and angle " $\theta$. "

\subsection{Transformations}

In order to arrange series of isosceles trapezoids into patterns, shape rules are developed based on observations of relationships between isosceles trapezoids in found Otavalo textiles. These rules are shown graphically in Figure 5. Rules are organized according to basic shape transformations; move, mirror, rotate, and scale. 

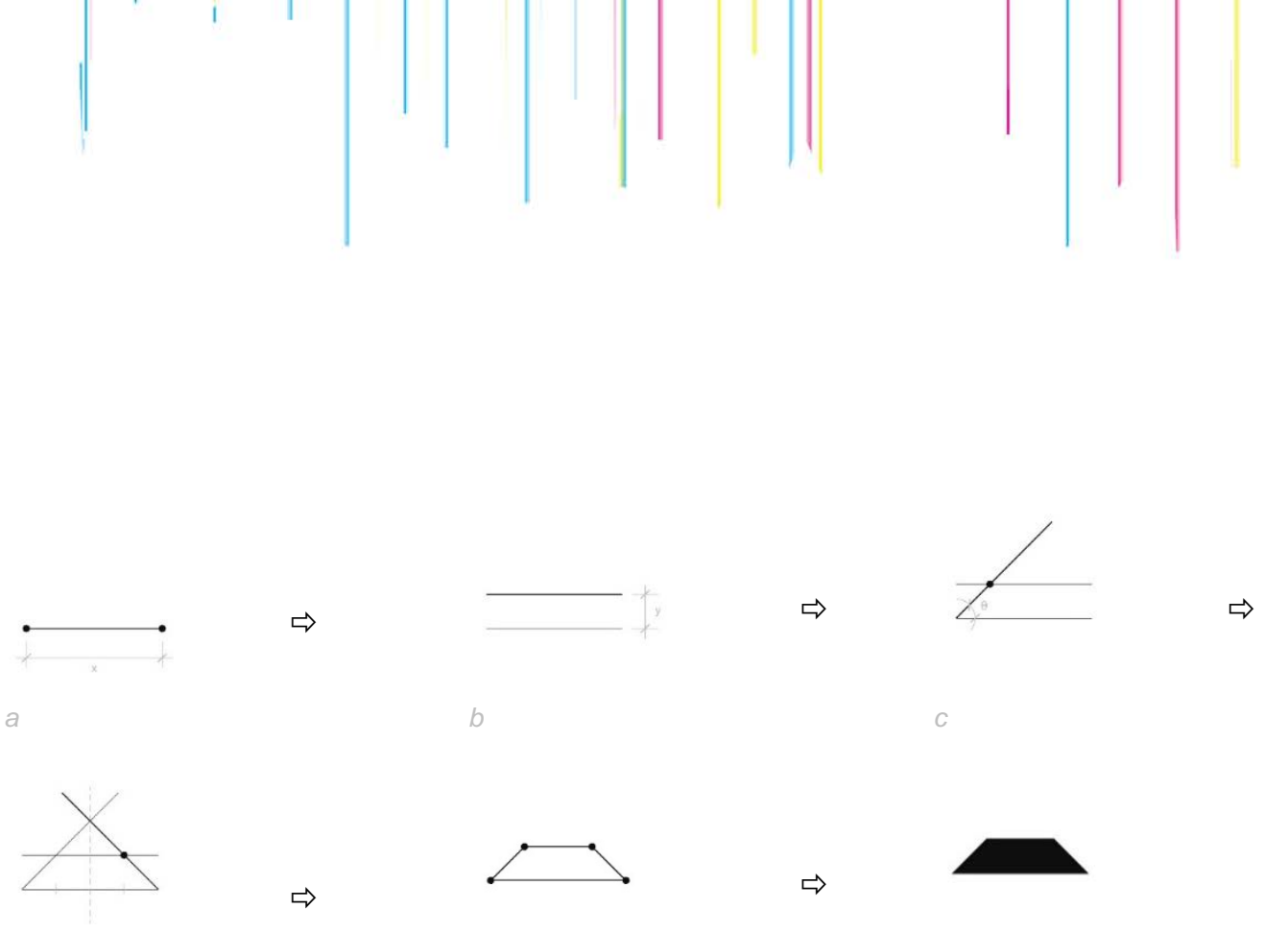

Figure 4. Isosceles trapezoid construction.

Rule 0 creates an initial shape. Using Rule 1a, a copy of the initial shape is moved horizontally by an increment equal to the shape width. Whereas using Rule $1 b$, a copy of the initial shape is moved horizontally by a variable amount.

Using Rule 2a, a copy of the initial shape is moved vertically by an increment equal to the shape height. Whereas using Rule $2 b$, a copy of the initial shape is moved vertically by a variable amount.

Using Rule $3 a$, a copy of the initial shape is reflected about the bottom edge of the shape. Whereas using Rule 3b, a copy of the initial shape is reflected about the bottom edge of the shape and moved vertically by a variable amount. Using Rule $4 a$, a copy of the initial shape is reflected about the top edge of the shape. Whereas using Rule $4 \mathrm{~b}$, a copy of the initial shape is reflected about the top edge of the shape and moved vertically by a variable amount. Certain variables used in Rules $3 \mathrm{~b}$ or $4 \mathrm{~b}$ will have the same result.

Using Rule 5, a copy of the initial shape is rotated about a defined vertex by an angle. Using Rule 6, a copy of the initial shape is shifted horizontally and vertically by variable amounts. Observed in found Otavalo textiles, these variables are often $1 / 2 x$ (one half of the shape length) horizontally and $y$ (equal to the shape height) vertically.

Using Rule 7, a copy of the initial shape is scaled about a defined vertex by an amount larger than 1 . Whereas using Rule 8 , a copy of the initial shape is scaled about a defined vertex by an amount larger than 0 and less than 1 .

Using Rule 9, a given shape can be substituted with a parametric variation of the shape. Rule 10 allows a selected shape to be removed. 


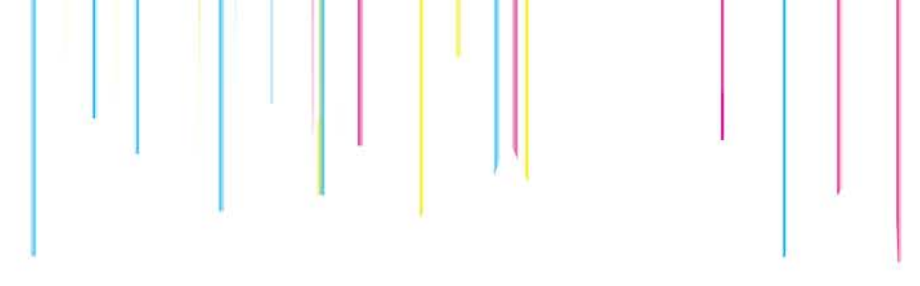

\subsection{Production 2: Array}

Figure 7 demonstrates the production of an arrayed pattern using Rules 1a and $2 a$, distributing a series of shapes across a field horizontally by an increment equal to the shape width (Rule 1a) and vertically by an increment equal to the shape height (Rule 2a). First, Rule 1a is instantiated three times to create the first row. Then, Rule $2 a$ is applied to each isosceles trapezoid in the first row to create the second row. Rule $2 a$ is repeated to produce the desired number of rows.

Alternatively, the resulting pattern in Figure 7 could be replicated and described as: First, Rule $2 \mathrm{a}$ is instantiated thirteen times to create the first column. Then, Rule $1 \mathrm{a}$ is applied to each isosceles trapezoid in the first column to create the second column. Rule 1a is repeated to produce the desired number of columns.

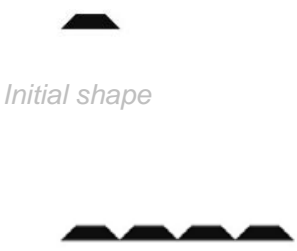

Rule $1 \mathrm{a}$

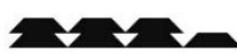

Rule $2 a$

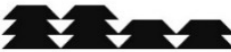

$\Rightarrow$

$\Rightarrow$

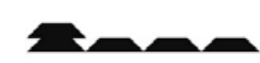

Rule $2 a$

$\Rightarrow$

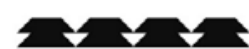

Rule $2 a$

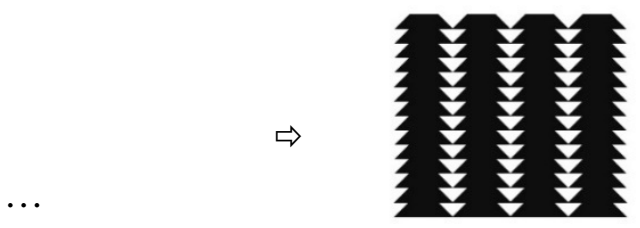

$\Rightarrow$

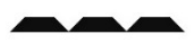

Rule $1 \mathrm{a}$

$\Rightarrow$

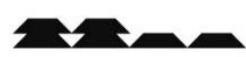

Rule $2 \mathrm{a}$

$\Rightarrow$

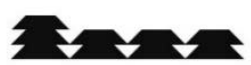

$\Rightarrow$

Figure 7. Arrayed pattern production. 


\section{Creating new patterns}

The process shown in Figure 7 can be replicated using linear array commands. First, the isosceles trapezoid is arrayed four times horizontally by an increment equal to the shape width. Then, this row is arrayed fourteen times vertically by an increment equal to the shape height. (Again, the described order of operations could be flipped; columns then rows.)

As our isosceles trapezoid shape definition, shape rules, and array distributions are created in a linked parametric model, an enormous variety of patterns can be quickly generated and studied. Patterns using additional shape configurations, shape rules, and combinations are illustrated in Figures 8 and 9.

Figures $8 \mathrm{a}$-i use Rules $1 \mathrm{a}$ and $2 \mathrm{a}$, demonstrating variations in patterning based on adjustments to shape width, height, and angle. Figures 8j-r use Rule $1 \mathrm{~b}$ and $2 \mathrm{~b}$, varying the amount shapes are moved horizontally and vertically. Figures 9a-f use Rule $3 a, 3 b, 4 a$, and $4 b$, demonstrating patterns with mirrored shape relationships. Figures $9 \mathrm{~g}-\mathrm{I}$ use variants of Rule 5 , demonstrating patterns with rotated shape relationships. Figures $9 \mathrm{~m}-\mathrm{o}$ use Rule 6 , demonstrating patterns with shifted shape relationships. Figures 9p-r use variants of Rules 7 and 8 , demonstrating patterns with scaled shape relationships.

Figures 8c, 8f, 8i, 8l, 8o, 8r, 9c, 9f, 9i, 9l, 9o, and 9r represent anomalies; patterns which break certain rules (alternating rows or columns, non-isosceles trapezoid shapes, random reduce, etc.) but are nevertheless part of the Otavalo textile language.

Based on the above collection of patterns generated digitally, sets of physical models are fabricated to further explore the Otavalo textile shape rules and possible assemblies. Initial physical models remain two-dimensional. In addition to the isosceles trapezoid, other related shapes - triangle and rhombus, which appear as overlapping figures or void spaces in patterns illustrated in Figures 8 and 9 - are also included. Sets of these parts are provided to study participants along with a description of shape rules. Both "solid" and "frame" shapes are represented. Participants are able to use these components to visualize and explore shape rules and develop existing and new patterns more readily with these physical models than digital models (a la Froebel blocks (Stiny, 1980)).

Some students focus on following shape rules, others create departures (shape orientation, reflected stacked rows, diagonal emphasis). Information gathered informs model iterations, shape rules, and example patterns depicted in Figures 8 and 9. 

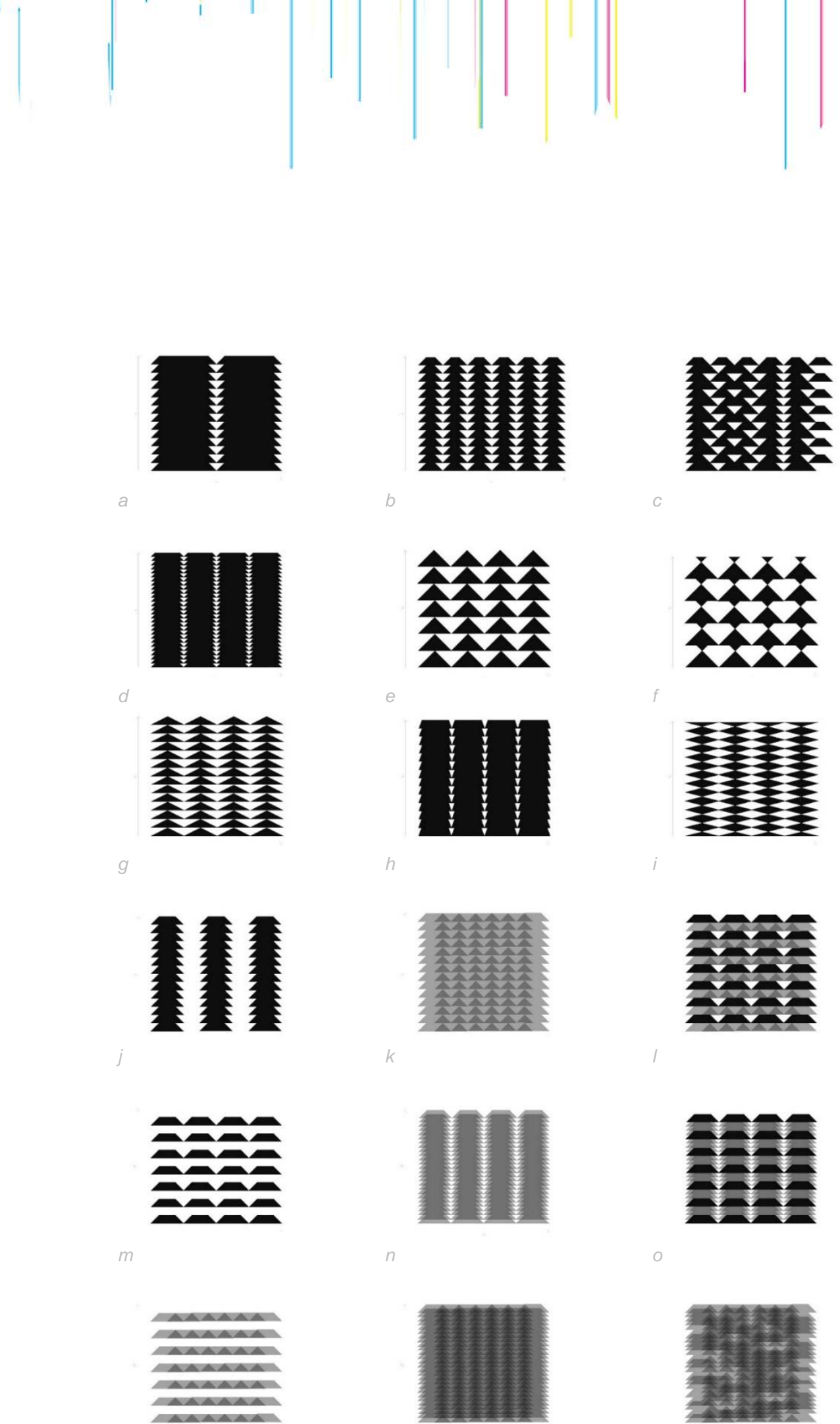

Figure 8. Patterns generated using the Otavalo Textile Grammar. 

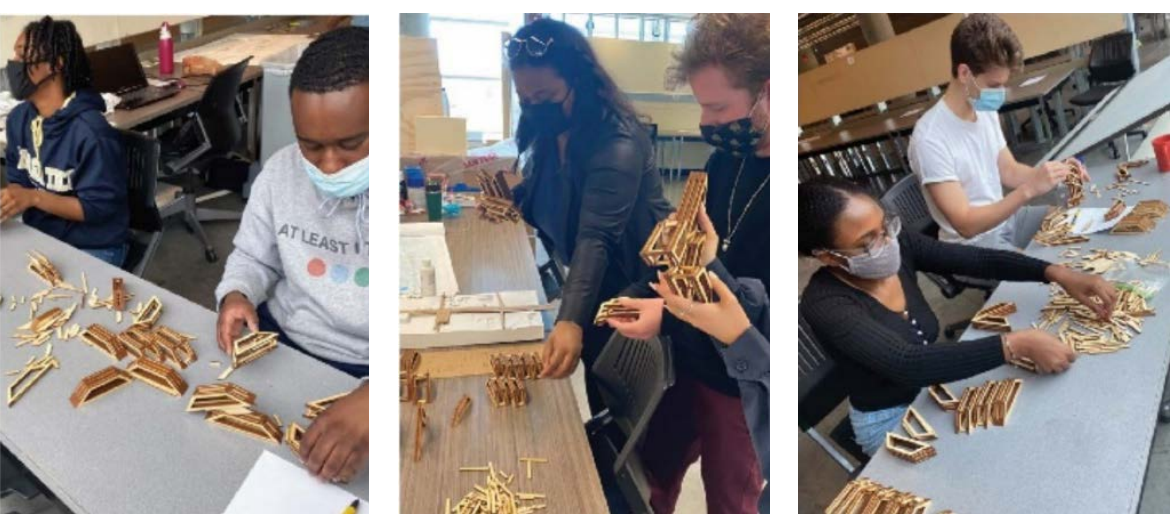

Figure 10. Student participants building with spaceframe modules.

\section{$4 \quad$ Creating new dialogues}

A three-dimensional model is developed and prototyped in order to explore spatial possibilities emanating from the Otavalo Textile Grammar. Modules are composed of laser cut plywood parts assembled into an extruded isosceles trapezoid spaceframe. Design variations studied included extrusion depths, number of layers, and embedded shapes (scaled isosceles trapezoids).

A number of these new building blocks are provided to study participants along with a description of shape rules; a reconsideration of ordinary ways of making. Module assembly sessions are conducted in groups, encouraging students to interact, discuss, and compare constructs; Figure 10.

Group modelling sessions also offer students the opportunity, if comfortable, to share their personal stories; indeed, to share stories of difference.

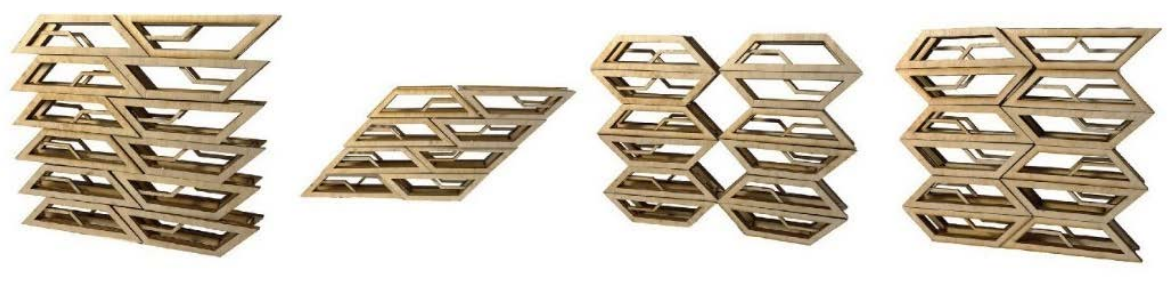

Figure 11. Example physical constructs. 


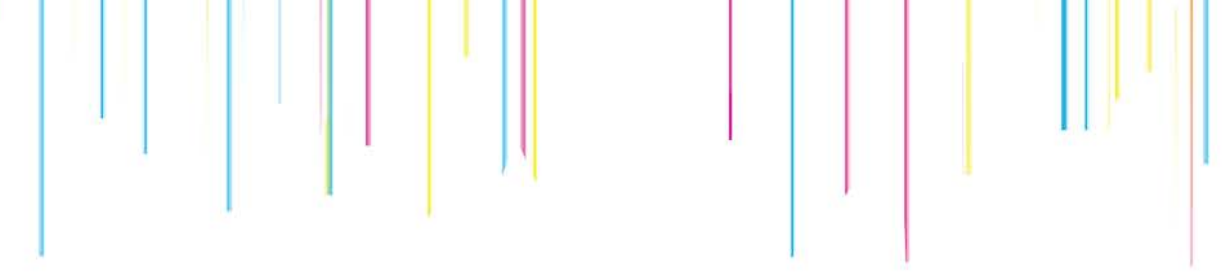

Completed models - examples of which are shown in Figures 11 and 12 - are analyzed, assembly rules recorded, and pattern syntax defined. Some models exhibit shape rules while others find novel ways for isosceles trapezoid modules to assemble; repetitive stacks, cantilevered elements, various module alignments and densities. Photographs of additional models - shown in Figure 12 - are used to aggregate further patterns; evoking previous two-dimensional physical and digital work.

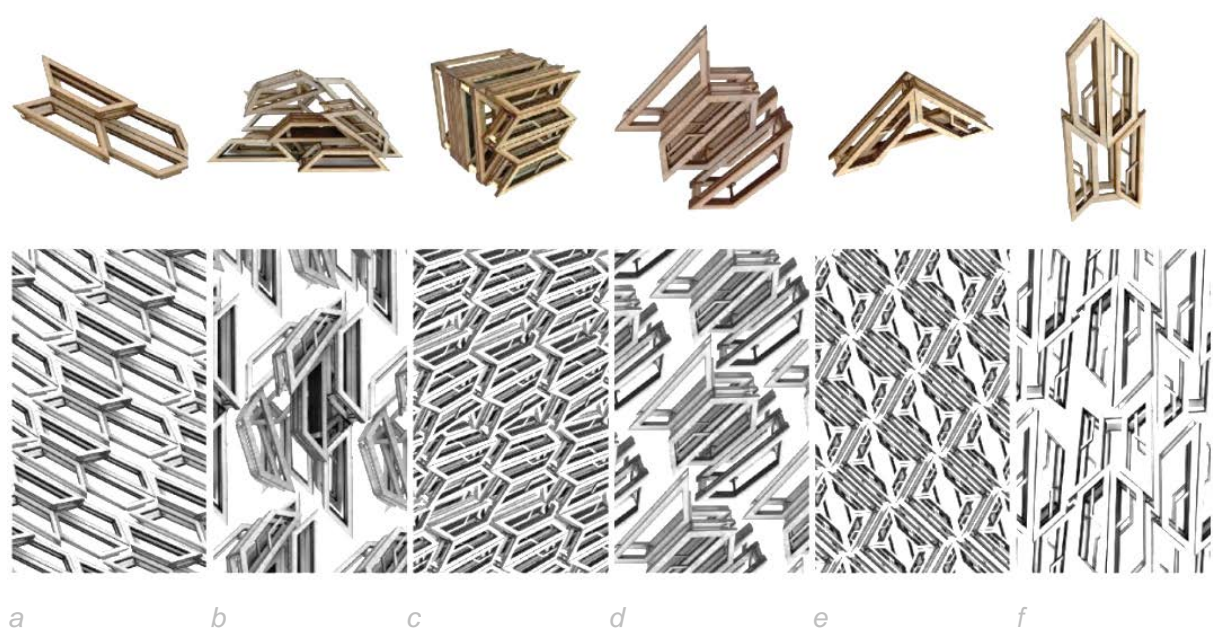

Figure 12. Additional physical constructs and corresponding novel patterns.

\section{Discussion}

As the Otavalo Textile Grammar definition and subsequent digital and physical experiments began as a case study for incorporating inspiration from cultural and textile references more meaningfully into architectural design, there is more work to do. This work has focused on one culture, one artifact, and mostly on one shape. There are many more to study and share; more geometry, more textiles, and more stories.

Still, within the context of this study, additional work could investigate effects of the weaving process itself on resultant patterns, limiting shape rules. More iterations of spatial physical models could be developed at defined architectural scales; anomalies defined, proportions considered. If implemented as building facades, environmental performance simulation of various patterns could be conducted. This study has also not yet incorporated analysis of the use and effects of color in found Otavalo textiles. 
Weston, Rourke Brakeville, Sally Murtadhi, Ruthana Leafy, Austin Turner, and Renee Palmer.

\section{References}

Anzaldua G. Borderlands/La Frontera: The New Mestiza. Aunt Lute Books. 1987.

April J. In Ecuador, Andean Artisans Are Using the Internet To Save Their Pre-Incan Traditions. Culture Trip. February 13 2020. https://theculturetrip.com/southamerica/ecuador/articles/in-ecuador-andean-artisans-use-the-internet-to-savetheir-pre-incan-traditions/

Delgado M. Architectural + Language: Breaking barriers and creating cultural dialogue. Bachelor of Architecture Theses, 5th Year. 2021.

https://digitalcommons.kennesaw.edu/barch_etd/164

Depositphotos. Quasarphoto. 2021. https://depositphotos.com/136371578/stockphoto-colourful-textiles-in-otavalo.html

Etsy. Hippy Happy Shop. 2021. https://www.etsy.com/listing/1013232578/southamerican-tapestry-ecuador-otavalo

Fashion Pachanga. INSPIRATION: PATTERNS FROM THE ANDES. 2021. https://fashionpachanga.wordpress.com/2013/02/13/inspiration-patterns-from-theandes/

Low S, Smith N. The Imperative of Public Space. Public Space Reader. Routledge. 2021.

Noel V. The Bailey-Derek Grammar: Recording the Craft of Wire-Bending in the Trinidad Carnival. Leonardo. Volume 48. Issue 4. Pages 357-365. 2015.

Pixabay. DEZALB. 2021. https://pixabay.com/photos/ecuador-otavalo-market-fabric$1257126 /$

Postrel V. The Fabric of Civilization: How Textiles Made the World. Basic Books. 2020.

Rowe A P, Miller L M, Meisch L A. Weaving and Dyeing in Highland Ecuador. University of Texas Press. 2007.

Stiny G. Ice-ray: a note on the generations of Chinese lattice designs. Environment and Planning B. Volume 4. Pages 89-98. 1977.

Stiny G. Kindergarten Grammars: Designing with Froebel's Buildings Gifts. Environment and Planning B. Volume 7. Pages 409-462. 1980.

Stiny G, Mitchell W J. The grammar of paradise: on the generation of Mughul gardens. Environment and Planning B. Volume 7. Pages 209-226. 1980.

Ucros C. The City of Otavalo: Between Reason And Magic. Culture Trip. December 12 2015. https://theculturetrip.com/south-america/ecuador/articles/the-city-of-otavalobetween-reason-and-magic/ 